\title{
ANÁLISE DAS RELAÇÕES ENTRE CRESCIMENTO ECONÔMICO E EMISSÕES DE GASES DE EFEITO ESTUFA NA AMÉRICA LATINA
}

\author{
Helson Gomes de Souza * \\ Francisco José Silva Tabosa ${ }^{\dagger}$
}

\begin{abstract}
Resumo
O presente trabalho busca verificar a veracidade da hipótese ambiental de Kuznets para o crescimento econômico total e aplicado ao setor agrícola dos países da América Latina. Para tanto foram utilizados dados dispostos entre 1990 e 2014 . Utilizou-se uma metodologia capaz de incorporar os efeitos da cointegração e da não estacionariedade. Os resultados permitiram concluir que as emissões de poluentes e o crescimento econômico formam uma relação no formato de ' $N$ '. Verificou-se também que os países da América Latina possuem incentivos a aumentarem suas emissões de poluentes em vista do crescimento econômico proporcionado. Concluiu-se também que no longo prazo o crescimento econômico exige maiores níveis de degradação ambiental do que nos estágios iniciais de crescimento.
\end{abstract}

Palavras-chave: Curva de Kuznets Ambiental, crescimento econômico, economia ambiental.

\begin{abstract}
The present work seeks to verify the veracity of the Kuznets' environmental hypothesis for total economic growth and applied to the agricultural sector of the countries of Latin America. For this purpose, data from 1990 to 2014 were used. A methodology to incorporate the effects of cointegration and non-stationarity was used. The results showed that pollutant emissions and economic growth form an ' $\mathrm{N}$ '-shaped relationship. It was also found that Latin American countries have incentives to increase their pollutant emissions in view of growth. It was also concluded that in the long run economic growth requires higher levels of environmental degradation than in the initial stages.
\end{abstract}

Keywords: Environmental Kuznets Curve, economic growth, environmental economics.

JEL classification: Q01, Q10, Q15.

DOI: http://dx.doi .org/10.11606/1980-5330/ea146724

\footnotetext{
* Graduado em Ciências Econômicas; Mestre em Economia Rural (PPGER-UFC); Doutorando em Economia Aplicada (PPGE-UFPB).E-mail: helson.g.souza@gmail.com

${ }^{\dagger}$ Doutor em Economia Aplicada (CAEN-UFC); Professor do Departamento de Economia Agrícola da Universidade Federal do Ceará (DEA-UFC). E-mail: franzetabosa@ufc.br
} 


\section{Introdução}

Os impactos ambientais causados pelo processo de crescimento econômico têm sido um assunto amplamente discutido nos últimos anos. Busca-se nos estudos efetuados acerca desse tema medir o real impacto do crescimento sobre o meio ambiente, para que, a partir dessa conclusão, seja possível questionar sobre como crescer reduzindo os impactos ambientais negativos dos processos de crescimento.

Jardón et al. (2017) demonstram que, nos países em processo de desenvolvimento, os impactos do crescimento econômico sobre o meio ambiente tendem a ser mais intensos, tendo em vista os valores das taxas de crescimento por eles alcançadas. Mas, especificamente em relação à América Latina, Jardón et al. (2017) ainda identificam que, no curto prazo, a elevação do crescimento econômico dos países gera aumentos na degradação ambiental, fato que não recebe a devida importância, tendo em vista a necessidade do crescimento para a busca de um melhor status de desenvolvimento socioeconômico nesses países.

Para Blanco et al. (2013), conhecer os impactos ambientais gerados pelo crescimento econômico nos países da América Latina seria um passo importante para o desenvolvimento de políticas públicas que visem a obtenção de um processo de crescimento com maior eficácia no que tange ao fator ambiental. Para os autores descritos, uma das principais implicações da degradação ambiental gerada pelo crescimento desses países está no elevado nível de emissões de gases de efeito estufa, o que, no longo prazo, poderia gerar impactos negativos sobre o desenvolvimento econômico dessas áreas, uma vez que o crescimento econômico depende das boas condições dos recursos naturais disponíveis.

De acordo com a conclusão encontrada por Zakarya et al. (2015), existe uma causalidade unidirecional significativa entre as emissões de gases de efeito estufa e os fatores determinantes do crescimento econômico nos países subdesenvolvidos. Existe, portanto, uma probabilidade de que os impactos ambientais causados pelo crescimento econômico atual nesses países interfiram de maneira negativa no seu processo de desenvolvimento socioeconômico futuro.

De acordo com Almeida et al. (2017), os impactos ambientais do crescimento econômico apresentam um comportamento temporal previsível, o qual é afetado por fatores ligados ao próprio crescimento econômico. Para os autores, uma das maneiras mais comuns e eficientes de obter essa tendência temporal é por meio da verificação da versão ambiental da hipótese postulada por Kuznets (1955). No entanto, Zafeiriou et al. (2017) destacam que, no setor agrícola, o ponto de inflexão do ' $U$ ' invertido proposto por essa hipótese pode demandar mais tempo para ocorrer, ou ocorre em um ponto de emissões superior a outros setores.

Tendo em vista essas considerações, o presente trabalho parte da hipótese de que as relações entre as emissões de poluentes e o crescimento econômico na América Latina seguem um comportamento temporal condizente com a hipótese ambiental do pressuposto de Kuznets (1955), e que na agricultura essa hipótese atesta um maior incentivo à emissão de poluentes.

Sendo assim, busca-se, por conseguinte, responder à seguinte problemática: Quais os impactos do crescimento econômico nas emissões de gases de efeito estufa dos países latino-americanos? E em que esses impactos diferem entre a economia como um todo e o setor agrícola? Nesse sentido, o presente 
estudo tem como objetivo verificar a hipótese ambiental de Kuznets para o crescimento econômico total e aplicado ao setor agrícola dos países da América Latina.

Para tanto, o presente trabalho é subdividido em cinco seções, incluindo esta breve introdução. A segunda, que se segue, engloba o embasamento teórico e literário sob o qual o trabalho encontra-se fundamentado. A terceira refere-se ao arcabouço metodológico utilizado. A quarta discute os resultados encontrados e as discussões construídas sobre o tema. Por fim, têm-se as considerações finais.

\section{Revisão de Literatura}

Estudos que investigam os efeitos do crescimento econômico na conjuntura ambiental relacionada às emissões de gases de efeito estufa são comumente vistos na literatura econômica. Dentre as abordagens utilizadas para investigação desse tema, a que melhor representa uma solução para os questionamentos estudados é a versão ambiental para o método proposto por Kuznets (1955). Essa abordagem é utilizada, por exemplo, nos estudos desenvolvidos por Luzzati \& Orsini (2009), Almeida et al. (2017), Kang et al. (2016), Jalil \& Mahmud (2009) e Sinha \& Shahbaz (2018).

No estudo desenvolvido por Sinha \& Shahbaz (2018), os autores buscam analisar os impactos ambientais da implementação de energias renováveis. No referido estudo, a versão ambiental para a Curva de Kuznets (CKA) é tratada como ferramenta mais adequada para responder os problemas questionados. Por meio dessa ferramenta, Sinha \& Shahbaz (2018) concluem que a implementação de fontes renováveis de energia tem diferentes impactos em setores econômicos distintos. Assim, os autores citam a importância da aplicação de políticas públicas que associem o crescimento econômico à qualidade do meio ambiente.

A CKA também é utilizada no estudo de Almeida et al. (2017), onde buscouse verificar os impactos do crescimento econômico no meio ambiente. Dentre as conclusões possibilitadas pela CKA, os referidos autores puderam concluir que uma política pública fundamentada apenas no crescimento econômico não é capaz, por si só, de reduzir a degradação ambiental. Almeida et al. (2017) ainda destacam a necessidade da criação de um quadro de política ambiental consistente, coerente e eficaz, sendo que tal fato seria essencial para melhorar a qualidade ambiental, a qual fornece melhorias de bem-estar e permite o desenvolvimento econômico a longo prazo.

Nasir \& Rehman (2011) utilizam a CKA para investigar a relação entre as emissões de $\mathrm{CO}_{2}$, renda, consumo de energia e comércio exterior no Paquistão, considerando o período de 1972-2008. Os autores concluem, a partir do método utilizado, que existe uma relação de longo prazo quadrática entre as emissões de $\mathrm{CO}_{2}$ e a renda, confirmando a existência da Curva Ambiental de Kuznets para a área utilizada. Os autores ainda destacam o fato de que, para o curto prazo, o crescimento econômico provindo das atividades analisadas proporciona alterações positivas nas emissões de gases de efeito estufa, o que geraria uma redução de curto prazo no bem-estar social provindo da degradação ambiental.

Jardón et al. (2017) verificam a veracidade da hipótese de Kuznets aplicada ao fator ambiental em um estudo voltado para as emissões de $\mathrm{CO}_{2}$ nos 
países da América Latina. Os autores afirmam que a verificação da hipótese é bastante sensível às mudanças estruturais súbitas. Para tanto, os autores consideram que as estimações convencionais utilizando dados em painel podem não representar o verdadeiro formato da CKA. Com isso, é sugerido no referido estudo que nesse tipo de investigação deve-se tomar os procedimentos econométricos satisfatórios para a correção de problemas advindos do comportamento temporal dos dados, como os problemas de raiz unitária e cointegração.

Al-Mulali et al. (2015) parte desse mesmo pressuposto e examina a veracidade da hipótese ambiental de Kuznets para o Vietnam por meio de uma metodologia Autoregressive Distributed Lag (ARDL). Os autores não confirmam a existência do ' $U$ ' invertido, uma vez que comprovam que no país em questão o crescimento econômico impacta positivamente as emissões de poluentes tanto no curto prazo quanto no longo prazo. Al-Mulali et al. (2015) ainda determinam que a entrada de capitais e as importações de bens e serviços atuam como fatores determinantes das elevações nas emissões de gases de efeito estufa no país, fornecendo indícios da necessidade de uma medida de controle de poluentes decorrentes desses fatores.

Zafeiriou et al. (2017) utilizam a CKA para analisar os efeitos do crescimento econômico para com o meio ambiente em três países da União Europeia. Os autores verificam que a hipótese ambiental de Kuznets é confirmada a longo prazo para dois dos três países, enquanto a curto prazo é validada em apenas um dos países. Os resultados encontrados pelos referidos autores ainda fornecem indícios de que a adoção de práticas agrícolas favoráveis ao meio ambiente e seleção de cultivos não garante simultaneamente altos desempenhos econômico e ambiental, pelo menos a curto prazo para os países estudados. Assim, os referidos autores enfatizam a importância da execução de medidas que forneçam ao produtor rural a possibilidade de trabalhar de maneira a reduzir os impactos ambientais das suas práticas trabalhistas.

De acordo com o que foi exposto no estudo desenvolvido por Khashman et al. (2016), os impactos ambientais gerados pelo setor agrícola ocorrem em uma escala superior a grande parte dos setores econômicos ${ }^{1}$. Com isso, os autores evidenciam a necessidade de adaptação da agricultura às novas técnicas de produção que associem os altos níveis tecnológicos aos baixos níveis de degradação ambiental.

Estudos como o que foi desenvolvido por Vlontzos et al. (2017) afirmam que os níveis de emissões de poluentes no setor agrícola estão diretamente associados ao aparato tecnológico e à eficiência técnica dos produtores agrícolas. Os referidos autores utilizam a CKA associada a um método de Análise Envoltória de Dados (DEA) para verificação desse contexto. Com isso, Vlontzos et al. (2017) destacam a necessidade do aprimoramento tecnológico do setor agrícola e a melhora nos níveis de eficiência técnica e tecnológica dos produtores rurais.

Martinho (2015) afirma que um crescimento econômico compatível com uma boa qualidade do meio ambiente será uma meta difícil de ser alcançada no futuro, seja nos países desenvolvidos, seja nas economias emergentes. $\mathrm{O}$ autor verifica que o crescimento econômico ocorre de maneira mais intensa com

\footnotetext{
${ }^{1}$ Outros estudos também demonstram a existência dessa característica. Qiao et al. (2019), por exemplo, indicam que a agricultura é o segundo maior emissor mundial de gás de efeito estufa devido ao uso de fertilizantes à base de combustíveis fósseis, maquinário agrícola e queima de biomassa.
} 
maiores níveis de degradação ambiental ${ }^{2}$. Com isso, dada a importância do crescimento, principalmente para os países em processo de desenvolvimento, seria normal que o crescimento econômico exigisse cada vez mais do meio ambiente, gerando, com isso, elevadas perdas da qualidade ambiental, o que, posteriormente, acarretaria uma perda generalizada de bem-estar social.

Tratando especificamente dos países da América Latina e Caribe, Al-Mulali et al. (2015) identificam ganhos marginais do crescimento econômico provindos da degradação ambiental. Por meio da estimação de uma CKA, os autores demonstram a necessidade de investimento em tecnologias que possibilitem o crescimento econômico associado a menores impactos no meio ambiente. Os autores citam as possibilidades de investimento em fontes de energia limpa e a necessidade de adaptação de setores como a agricultura às tecnologias menos agressivas ao meio ambiente.

Nas economias emergentes, é comum que as atividades provindas do setor primário tenham grande participação na composição do crescimento. As commodities agrícolas, em muitos casos, movimentam uma boa parte do processo de crescimento econômico. Essas atividades, contudo, auferem altos níveis de impacto ambiental, advindos da exploração dos recursos e consequências indiretas como as emissões de poluentes e a perca de qualidade de vida e de bem-estar social. Elevar o crescimento econômico nesses países pode gerar consequências alternadas a depender do estágio de curto ou longo prazo. Deve-se, portanto, buscar técnicas de crescimento que permitam o desenvolvimento das atividades primárias de forma a proporcionar menos consequências ambientais, seja no curto ou no longo prazo (Page et al. 2014).

Para sintetizar de uma forma a facilitar uma rápida compreensão e fornecer uma base literária sobre o assunto em questão, a Tabela 1 explana um conjunto de contribuições sobre os estudos das emissões de poluentes por meio da CKA. Nota-se que, nos casos considerados na Tabela 1, independente da área de análise, os estudos que consideraram a CKA na sua forma quadrática obtiveram um formato de ' $U$ ' invertido, enquanto os estudos que consideraram a forma cúbica se depararam com uma CKA na forma de ' $\mathrm{N}$ '.

Tabela 1: Contribuições literárias para a CKA

\begin{tabular}{l|c|c|c}
\hline Autor & Método & Área de aplicação & Formato \\
\hline Zafeiriou et al. (2017) & VECM & Agricultura & 'U' invertido \\
\hline Ben et al. (2017) & VECM & Energia & 'U' invertido \\
\hline Tao et al. (2008) & DOLS, FMOLS & Combustíveis & 'N' invertido \\
\hline Vlontzos et al. (2017) & $\begin{array}{c}\text { Painel } \\
\text { (erros robustos) }\end{array}$ & Agricultura & 'N' \\
\hline Ávila \& Diniz (2015) & DOLS, FMOLS & Emissões totais & 'N' \\
\hline Nasir \& Rehman (2011) & VECM & Emissões totais & 'U' invertido \\
\hline Apergis \& Ozturk(2015) & DOLS, FMOLS & Emissões totais & 'N' \\
\hline Jebli \& Youssef (2015) & VECM & Emissões totais & 'U' invertido \\
\hline Liu et al. (2017) & VECM & Agricultura & 'U' invertido \\
\hline Zhang et al. (2017) & VECM & Emissões totais & 'U' invertido \\
\hline
\end{tabular}

Fonte: Elaborado pelos autores.

\footnotetext{
${ }^{2}$ Essa característica provém da relação positiva entre crescimento e degradação ambiental proposta em literatura, como no caso da CKA, por exemplo, e é válida tanto para países desenvolvidos quanto para países em desenvolvimento.
} 


\section{Metodologia}

\subsection{Dados}

Para que se cumpra a proposta formulada anteriormente, o presente trabalho efetua estimações econométricas que consideram duas áreas diferenciadas, sendo uma estimação para o setor agrícola e outra estimação sem desagregação setorial dos dados. Para representar o crescimento econômico da agricultura, utilizou-se o somatório (em toneladas) da produção de algodão, arroz, café, açúcar, feijão, girassol, milho, mandioca, soja, sorgo, trigo, batata, abacaxi, coco, leite, carne e queijo, sendo esse valor dividido pela quantidade de habitantes com intuito de gerar uma aproximação para o nível de crescimento per capita desse setor ${ }^{3}$. Essas informações foram disponibilizadas na base de dados online da Comissão Econômica para a América Latina e o Caribe CEPAL (2018). Como uma medida de crescimento econômico que inclua todos os setores, utilizou-se o PIB per capita dos países latino-americanos (em US\$ de 2010).

Para fornecer uma medida para a degradação ambiental, foram utilizadas as emissões de $\mathrm{CO}_{2}$ per capita (toneladas/habitante), as quais foram extraídas da base de dados da CEPAL (2018). As emissões referentes ao setor agrícola consistem no montante de $\mathrm{CO}_{2}$ provindo da fermentação entérica, do maneio de estrume, do cultivo de arroz, do uso de fertilizantes sintéticos, do uso de adubo aplicado em solos e pastagens, dos resíduos de culturas agrícolas, do cultivo de solos orgânicos, dos resíduos de culturas incendiadas e das queimadas. Já as emissões totais consistem no somatório das emissões setoriais disponibilizadas pela CEPAL (2018). Já as emissões totais correspondem ao montante de $\mathrm{CO}_{2}$ emitido por todos os setores econômicos dos países aqui considerados.

Foram utilizados dados anuais dispostos entre 1990 e 2014. Escolheu-se esse período em vista da disponibilidade de dados para todas as variáveis estudadas. As informações comportam dados sobre 32 países distribuídos entre a América do Sul, Central e Caribe, além do México. A fonte de dados foi escolhida em vista de uma maior disponibilidade acerca das informações referentes ao setor agrícola. Além disso, o período estudado foi escolhido em vista da disponibilidade temporal dos dados desta fonte para os dois casos considerados neste trabalho.

\subsection{Tratamento econométrico}

Nesta investigação, a hipótese da CKA é analisada por meio de uma função logarítmica ou cúbica do PIB per capita como resposta às emissões de $\mathrm{CO}_{2}$ por habitante nos países latino-americanos. Adotou-se então uma especificação econométrica para dados em painel, assim como delimitado por Tao et al. (2008), a qual segue a forma:

\footnotetext{
${ }^{3}$ Ressalta-se que muitos trabalhos utilizam o PIB como medida de crescimento, no entanto, o PIB do setor agrícola não foi utilizado em vista da indisponibilidade de informações para uma quantidade relevante de países.
} 


$$
\begin{gathered}
\ln \left(\frac{E}{P}\right)_{i t}=\alpha_{i}+\theta_{t}+\beta_{1} \ln \left(\frac{P I B}{P}\right)_{i t}+\beta_{2}\left[\ln \left(\frac{P I B}{P}\right)_{i t}\right]^{2}+ \\
\beta_{3}\left[\ln \left(\frac{P I B}{P}\right)_{i t}\right]^{3}+\mu_{i t}
\end{gathered}
$$

onde $E$ representa as emissões de $\mathrm{CO}_{2}, P$ é a população do país $i$ no ano $t, P I B$ diz respeito ao Produto Interno Bruto total (ou PIB do setor agrícola), $\theta$ representa os efeitos do tempo específicos de cada período e $\mu$ é o erro aleatório.

Assim, consideram-se sete hipóteses neste trabalho, sendo elas: (i) $\beta_{1}>0$, $\beta_{2}<0$ e $\beta_{3}>0$, que confirma a forma de "N" no longo prazo Figura 1(a); (ii) $\beta_{1}<0, \beta_{2}>0$ e $\beta_{3}<0$, que indica uma forma de " $N$ " inverso Figura 1(b); (iii) $\beta_{1}<0, \beta_{2}>0$ e $\beta_{3}=0$, que denota uma forma de "U" normal Figura $1(\mathrm{c})$; (iv) $\beta_{1}>0, \beta_{2}<0$ e $\beta_{3}=0$, que revela a forma tradicional de " $U$ " invertido Figura $1(\mathrm{~d})$; (v) $\beta_{1}>0, \beta_{2}=0$ e $\beta_{3}=0$, que representa uma relação linear monotonicamente crescente Figura $1(\mathrm{e})$; (vi) $\beta_{1}<0, \beta_{2}=0$ e $\beta_{3}=0$, , a qual denota a existência de uma relação linear decrescente Figura 1(f); e (vii) $\beta_{1}=0$, $\beta_{2}=0$ e $\beta_{3}=0$, reveladora de uma relação perfeitamente elástica Figura $1(\mathrm{~g})$.

Figura 1: Representações gráficas das hipóteses da Curva de Kuznets Ambiental
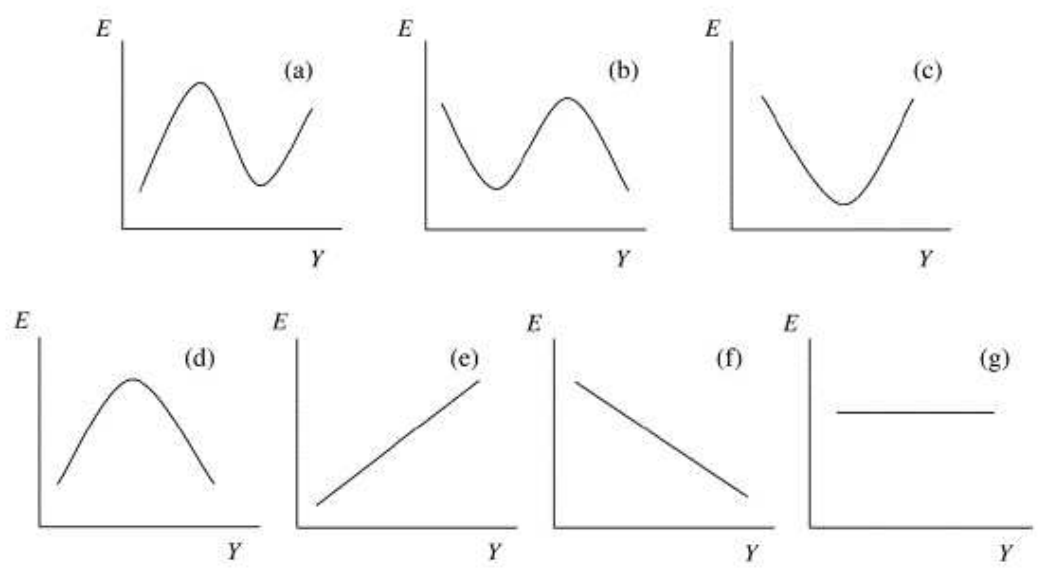

Fonte: Elaborado pelos autores com base em Tao et al. (2008).

Os dados dispostos no tempo, contudo, necessitam de um tratamento adequado, de maneira que torna-se necessária a averiguação de características específicas dessas séries. O problema de não estacionariedade ou raiz unitária é uma característica de dados com distribuições contidas no tempo. De acordo com Bueno (2008), a estacionariedade ocorre quando uma série flutua em torno de uma média fixa e se a variância dessa série é constante ao longo do tempo. Além disso, Bueno (2008) destaca que a constatação da estacionariedade é fundamental para que se permita proceder às inferências estatísticas sobre os parâmetros estimados com base na realização de um processo estocástico. Nesse sentido, antes de efetuar qualquer procedimento estatístico, é necessário que seja verificada a condição de estacionariedade dos dados. 
Considerando o fato de que alguns testes de estacionariedade de primeira geração consideram a hipótese de independência individual, utiliza-se neste trabalho o teste proposto por Pesaran (2007). Nesse processo, o referido autor formula um teste adaptado para $N$ unidades crossectional e $T$ unidades de tempo utilizando um modelo linear dinâmico com heterogeneidade de acordo com:

$$
Y_{i, t}=\left(1-\delta_{i}\right) \mu_{i}+\delta_{i} Y_{i, t-1}+u_{i, t}, \quad i=1, \ldots, N, \quad t=1, \ldots, T
$$

dado os valores iniciais $Y_{i, 0}$ e um único fator estrutural para os distúrbios $u_{i, t}=\lambda_{i} f_{t}+e_{i, t}$. A partir de um conjunto de especificações matemáticas e estatísticas advindas da equação (2), Pesaran (2007) considera um teste IPS que consiste na média aritmética das estatísticas de Dickey-Fuller aumentada das unidades crossectional, de acordo com:

$$
\text { CIPS }=\frac{1}{N} \sum_{i=1}^{N} C^{N} A F_{i}
$$

onde $C A D F_{i}$ é a estatística Dickey-Fuller aumentada para a unidade crossectional $i$. Com isso, a utilização desse teste busca verificar a hipótese nula de ausência de estacionariedade nas séries estudadas. Assim, caso a hipótese nula seja rejeitada, os dados são não estacionários. ${ }^{4}$ Para um melhor detalhamento da condição de estacionariedade dos dados, utilizam-se também os testes propostos por Maddala \& Wu (1999) e efetua-se o teste proposto por Im \& Pesaran (2003).

Para prosseguir com as inferências necessárias para esse tipo de dados, deve-se também, averiguar a condição de cointegração dos dados utilizados. Faz-se uso, portanto, do teste de cointegração proposto por Pedroni (1999), o qual foi desenvolvido com intuito de averiguar a ausência de cointegração para painéis dinâmicos com regiões heterogêneas. Esse teste pode ser especificado como:

$$
y_{i t}=\alpha_{i}+\delta_{i t}+\theta_{t}+\beta_{1 i} x_{1 t}+\ldots+\beta_{m i} x_{i t}+\epsilon_{i t}
$$

onde $i=1, \ldots, n$ e $t=1, \ldots, t$ são os números de unidades de espaço e tempo, respectivamente, $m$ é a quantidade de regressores e $\alpha_{i}, \delta_{i t}$ e $\theta_{t}$ representam os efeitos individuais específicos, a tendência linear e os efeitos do tempo, respectivamente. A hipótese nula do teste indica a ausência de cointegração.

\subsection{Estimação}

A estimação utilizada é baseada na correção dos problemas de cointegração e raiz unitária para dados empilhados. Utilizou-se, portanto, o procedimento fully modified OLS (FMOLS) para correção de cointegração, que foi proposto por Phillips \& Moon (1999) e Pedroni (1999), além de ter sido utilizado o

\footnotetext{
${ }^{4}$ Mais detalhes em Pesaran (2007).
} 
processo Dynamic OLS (DOLS), proposto por Kao \& Chiang (2000), sendo ambos utilizado por Tao et al. (2008).

O estimador FMOLS modifica os mínimos quadrados para explicar os efeitos de correlação serial e de endogeneidade nos regressores que surgem da existência de uma relação de cointegração. Sua especificação pode ser dada pela forma:

$$
\hat{\beta}^{*}=N^{-1} \sum_{1}^{N}\left[\sum_{t=1}^{T}\left(x_{i t}-\hat{x}_{i}\right)^{2}\right]^{-1}\left[\sum_{t=1}^{T}\left(x_{i t}-\hat{x}_{i}\right) y_{i t}^{*}-T \hat{\gamma}_{i}\right]
$$

onde $y_{i t}^{*}=\left(y_{i t}-\bar{y}_{i}\right)-\frac{\hat{\Omega}_{21 i}}{\hat{\Omega}_{22 i}} \Delta x_{i t}$, sendo $\Omega_{i}$ o vetor de covariância de longo prazo, $\gamma_{i}=\hat{\Gamma}_{21 i}+\hat{\Omega}_{22 i}^{0}-\frac{\hat{\Omega}_{21 i}}{\hat{\Omega}_{21 i}}\left(\hat{\Gamma}_{22 i}+\hat{\Omega}_{22 i}^{0}\right)$ e $\hat{\beta}^{*}$ é o estimador FMOLS.

O estimador DOLS é paramétrico e fornece uma opção alternativa ao FMOLS. A representação matemática desse processo pode ser feita considerando uma regressão cointegrada para dados homogêneos em painel, como:

$$
\begin{gathered}
y_{i t}=\alpha_{i}+\lambda_{i t}+\theta_{t}+\beta^{\prime} x_{i t}+\mu_{i t} \\
x_{i t}=x_{i t-1+v_{i t}}
\end{gathered}
$$

sendo $i=1, \ldots, N$ e $t=1, \ldots, T$. $x_{i t}$ é um vetor $k x 1$ de regressores compensados. $\alpha_{i}, \lambda_{i t}$ e $\theta_{t}$ representam os efeitos específicos individuais, a tendência linear individual específica e os efeitos comuns do tempo, respectivamente. Nesse caso, as variáveis independentes são cointegradas, de ordem 1 para todo $i$, sendo estacionárias em primeira diferença. O estimador em questão baseia-se na decomposição do erro.

$$
\mu_{i t}=\sum_{j=-p}^{q} \gamma_{j}^{\prime} \Delta x_{i t-j}+\epsilon_{i t}
$$

onde $p$ e $q$ representam o número de observações e defasagens, respectivamente. Além disso, $\epsilon_{i t}$ é ortogonal para todas as observações e defasagens da primeira diferença dos regressores. Inserindo a equação (6) na equação (5), botem-se:

$$
y_{i t}=\alpha_{i}+\lambda_{i t}+\theta_{t}+\beta^{\prime} x_{i t} \sum_{j=-p}^{q} \gamma_{j}^{\prime} \Delta x_{i t-j}+\epsilon_{i t}
$$

Melhores especificações dos processos matemáticos de obtenção dos estimadores DOLS e FMOLS podem ser encontrados em Phillips \& Moon (1999), Pedroni (1999) e Doan \& Pedroni (2001).

\section{Resultados e Discussão}

Inicialmente, faz-se aqui uma breve análise dos dados utilizados por meio das estatísticas descritivas das variáveis incluídas nos procedimentos estatísticos 
posteriores. A Tabela 2 mostra as estatísticas descritivas das variáveis usadas nos dois painéis de dados, comportando a média, o desvio padrão e os valores máximo e mínimo para o agregado de dados de cada painel. O que se pode observar a partir das estatísticas descritivas é que, em temos médios, as emissões per capita provindas do setor agrícola são menores do que as emissões per capita totais, o que de fato deve acontecer.

Durante o período estudado, em média, o PIB per capita total foi de US\$ $6.630,00$ para os países estudados. Além disso, a média da produção per capita do setor agrícola indica que, em média, os países aqui considerados produziram cerca de 0,0013 toneladas per capita de produtos agrícolas durante os anos de 1990 a $2014 .{ }^{5}$. Já as emissões totais médias foram de aproximadamente 3,05 toneladas por habitante, enquanto as emissões médias do setor agrícola obtiveram um valor médio de 1,22 toneladas por habitante.

Tabela 2: Estatísticas descritivas das variáveis utilizadas

\begin{tabular}{cccccc}
\hline Variável & Observações & Média & D.Padrão & Mínimo & Máximo \\
\hline \multicolumn{6}{c}{ Dados gerais } \\
\hline$\frac{E}{P}$ & 800 & 3,0532 & 4,3362 & 0,0390 & 36,7866 \\
$\frac{P I B}{P}$ & 800 & $6.630,0660$ & $5.306,6850$ & 668,8980 & $32.080,1000$ \\
\hline \multicolumn{6}{c}{ Dados da Agricultura } \\
\hline$\frac{E}{P}$ & 800 & 0,0012 & 0,0013 & 0,0001 & 0,0077 \\
$\frac{P I B}{P}$ & 800 & 0,0013 & 0,0014 & 0,0001 & 0,0079 \\
\hline
\end{tabular}

Fonte: Elaborado pelos autores.

Para ter uma melhor compreensão do comportamento das emissões de $\mathrm{CO}_{2}$, a Figura 2 demonstra a quantidade de $\mathrm{CO}_{2}$ liberado pelos países da América Latina entre 1990 e 2014. Observa-se que as emissões apresentam um comportamento crescente, seja na agricultura, seja na economia como um todo. Essa elevação nas emissões de poluentes pode ser explicada pelo processo de crescimento econômico experimentado por esses países, o qual, ocorre de forma a provocar degradações ambientais, principalmente no setor agrícola.

Na Figura 2 também é possível visualizar a proporção das emissões de $\mathrm{CO}_{2}$ do setor agrícola em relação às emissões totais na América Latina. Nota-se que, fazendo uma comparação entre os períodos final e inicial, a agricultura reduziu sua participação no montante de emissões da economia latino-americana. No início do período analisado, as emissões provindas do setor agrícola representavam mais de $70 \%$ do total de $\mathrm{CO}_{2}$ nos países aqui estudados. Já em 2014 esse percentual cai para pouco mais de $50 \%$.

Nota-se que a agricultura possui uma elevada participação nos níveis de emissões da América Latina. Esse fato condiz com o que foi exposto por Page et al. (2014), onde é descrito que as atividades provindas do setor primário apresentam grande participação no crescimento econômico dos países subdesenvolvidos e em desenvolvimento.

Como os dados utilizados nas inferências estatísticas estão distribuídos ao longo do tempo, torna-se necessário verificar as características temporais da

\footnotetext{
${ }^{5}$ Cabe ressaltar que esses valores advêm de uma aproximação utilizando o somatório do peso da produção das culturas agrícolas.
} 
Figura 2: Dispersão das emissões ao longo dos anos
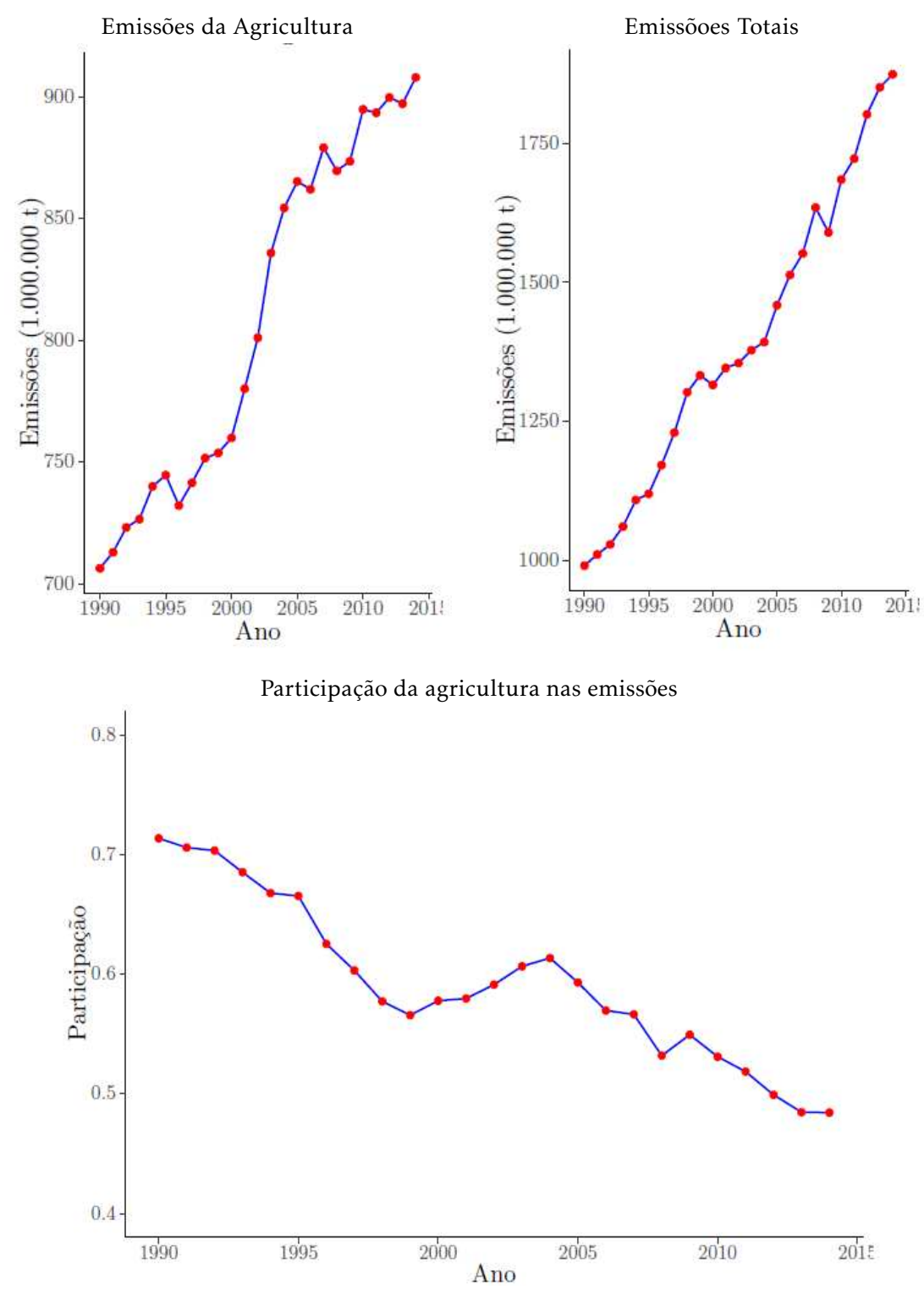

Fonte: Elaborado pelos autores. 
dispersão desses valores, assim como designado por Bueno (2008). A Tabela 3 apresenta os resultados dos testes de estacionariedade efetuados para as variáveis utilizadas em nível e em primeira diferença.

Para obter uma verificação mais detalhada da existência ou não da estacionariedade, efetuou-se o teste de Pesaran (2007) considerando a inclusão e ausência da tendência temporal.

O que se pode concluir a partir desse procedimento é que, tanto para os dados totais quanto para a agricultura, aceitou-se a hipótese nula de inexistência de estacionariedade. Quando efetuada a primeira diferença, contudo, a hipótese nula é rejeitada, atestando, com isso, a inexistência de raiz unitária na primeira diferença em ambos os casos.

Tabela 3: Resultados para o teste de estacionariedade de Pesaran (2007)

\begin{tabular}{|c|c|c|}
\hline \multicolumn{3}{|c|}{ Dados gerais } \\
\hline & Sem tendência & Com tendência \\
\hline$\left[\ln \left(\frac{E}{P}\right)\right]$ & $-1,029$ & $-1,318$ \\
\hline$\left[\ln \left(\frac{P I B}{P}\right)\right]$ & 0,805 & 1,000 \\
\hline$\left[\ln \left(\frac{P I B}{P}\right)\right]^{2}$ & 0,988 & 1,000 \\
\hline$\left[\ln \left(\frac{P I B}{P}\right)\right]^{3}$ & 1,195 & 1,000 \\
\hline$\Delta\left[\ln \left(\frac{E}{P}\right)\right]$ & $-20,307^{*}$ & $-18,770^{*}$ \\
\hline$\Delta\left[\ln \left(\frac{P I B}{P}\right)\right]$ & $-10,108^{*}$ & $-8,447^{*}$ \\
\hline$\Delta\left[\ln \left(\frac{P I B}{P}\right)\right]^{2}$ & $-10,049^{*}$ & $-8,382^{*}$ \\
\hline$\Delta\left[\ln \left(\frac{P I B}{P}\right)\right]^{3}$ & $-9,979^{*}$ & $-8,309^{*}$ \\
\hline \multicolumn{3}{|c|}{ Dados Agricultura } \\
\hline $\ln \left(\frac{E}{P}\right)$ & $-0,816$ & $-0,544$ \\
\hline$\left[\ln \left(\frac{P I B}{P}\right)\right]$ & 0,826 & $-0,341$ \\
\hline$\left[\ln \left(\frac{P I B}{P}\right)\right]^{2}$ & 0,601 & 0,218 \\
\hline$\left[\ln \left(\frac{P I B}{P}\right)\right]^{3}$ & 0,340 & 0,124 \\
\hline$\Delta\left[\ln \left(\frac{E}{P}\right)\right]$ & $-4,584^{*}$ & $-2,718^{*}$ \\
\hline$\Delta\left[\ln \left(\frac{P I B}{P}\right)\right]$ & $-6,839^{*}$ & $-4,759^{*}$ \\
\hline$\Delta\left[\ln \left(\frac{P I B}{P}\right)\right]^{2}$ & $-7,213^{*}$ & $-5,101^{*}$ \\
\hline$\Delta\left[\ln \left(\frac{P I B}{P}\right)\right]^{3}$ & $-7,415^{*}$ & $-5,249^{*}$ \\
\hline
\end{tabular}

Fonte: Elaborado pelos autores.

Nota: Valores sucedidos do símbolo * denotam a rejeição da hipótese nula de não estacionariedade a nível de $5 \%$ de significância estatística.

Verificada a ausência de estacionariedade nos dados, faz-se a seguir a verificação da existência de cointegração nos painéis utilizados. A Tabela 4 demonstra os resultados desse procedimento com base na utilização do teste de Pedroni (1999). A partir da estatística Augmented Dickey-Fuller (ADF), 
verifica-se que tanto para os dados com emissões totais quanto para os dados voltados para a agricultura, a existência de cointegração foi confirmada.

Em vista da não estacionariedade dos dados e da cointegração que foi verificada, as inferências estatísticas realizadas posteriormente devem considerar essa estrutura dos dados. Assim, a estimação da CKA será efetuada por meio do procedimento proposto por Phillips \& Moon (1999) e Pedroni (1999) e que foi utilizado em trabalhos como Tao et al. (2008).

Tabela 4: Resultados dos testes de cointegração

\begin{tabular}{c|ccc}
\hline \multicolumn{4}{c}{ Dados gerais } \\
\hline & Sem tendência & Com tendência & Sem constante \\
\hline Phillips-Perron modificado & $-0,2609$ & 1,2095 & $-0,7624$ \\
Phillips-Perron & $-7,4633^{*}$ & $-9,1309^{*}$ & $-8,3807^{*}$ \\
Dickey-Fuller aumentado & $-8,8286$ & $-9,6272^{*}$ & $-8,7211^{*}$ \\
\hline \multicolumn{4}{c}{ Dados da agricultura } \\
\hline Phillips-Perron modificado & $-8,2147^{*}$ & $-4,8866^{*}$ & $-9,6565^{*}$ \\
Phillips-Perron & $-21,9130^{*}$ & $-21,4691^{*}$ & $-19,1529^{*}$ \\
Dickey-Fuller aumentado & $-8,0758^{*}$ & $-8,1385^{*}$ & $-7,6633^{*}$ \\
\hline
\end{tabular}

Fonte: Elaborado pelos autores.

Nota: Valores sucedidos do símbolo * denotam a rejeição da hipótese nula de inexistência de cointegração a nível de 5\% de significância estatística.

Na Tabela 5, estão demonstrados os resultados encontrados com as estimações via Fully Modified OLS e Dynamic OLS. Para as estimações via DOLS, foram feitos testes para um conjunto de critérios de escolha de defasagem, assim como a ausência desses, de acordo com os mecanismos de escolha dispostos na literatura. As estimações que obedeceram os pressupostos indicados na literatura foram fundamentadas na ponderação de grupos (group mean), sendo que, para as estimações via DOLS, foram escolhidas as estimações com ausência de critérios de escolha de defasagens. Por meio desse procedimento, busca-se também controlar a heterogeneidade por duas vias, sendo a primeira, o fato de considerar os valores nas suas formas per capita, e a segunda, por meio dos efeitos fixos das estimações.

Os resultados demonstram que, tanto para as emissões totais quanto para as emissões do setor agrícola, as estimações via FMOLS não apresentaram significância estatística nos coeficientes, enquanto as estimações via DOLS apresentaram significância estatística para todos os coeficientes nos dois casos analisados. As distorções de resultados podem ser provindas da sensibilidade do estimador FMOLS ao tamanho dos grupos considerados na estimação, assim como da disparidade dos valores dentre as unidades de corte transversal utilizadas.

Os sinais dos coeficientes indicam a existência de uma CKA em um formato de ' $\mathrm{N}$ ', assim como descrito na Figura 1(a), seja para a economia como um todo, seja para o setor agrícola. Assim, o que se pode verificar a partir desse resultado é que, para os países da América Latina, a elevação do crescimento econômico, seja de uma forma geral na economia, seja no setor agrícola, gera inicialmente um aumento nos níveis de emissões de $\mathrm{CO}_{2}$, relação essa que passa a assumir um comportamento inverso com o decorrer do tempo. No longo prazo, contudo, um novo ponto de inflexão é verificado onde, a par- 
tir dele, o aumento do crescimento econômico volta a propiciar elevações nas emissões de poluentes.

Tabela 5: Resultados para as estimações DOLS e FMOLS

\begin{tabular}{c|cc|cc}
\hline & \multicolumn{2}{|c|}{ Dados gerais } & \multicolumn{2}{c}{ Dados da agricultura } \\
\hline & DOLS & FMOLS & DOLS & FMOLS \\
\hline $\operatorname{Ln}\left(\frac{P I B}{P}\right)$ & $2014,2800^{*}$ & $15,9600^{*}$ & $8,1400^{*}$ & 1,8300 \\
$\operatorname{Ln}\left(\frac{P I B}{P}\right)^{2}$ & $-302,2000^{*}$ & $-21,5400$ & $-2,8100^{*}$ & $-0,6400^{*}$ \\
$\operatorname{Ln}\left(\frac{P I B}{P}\right)^{3}$ & $10,1900^{*}$ & $1,1000^{*}$ & $0,0900^{*}$ & $-0,0500^{*}$ \\
\hline
\end{tabular}

Fonte: Elaborado pelos autores.

Nota: Valores sucedidos do símbolo * denotam significância estatística a nível de $5 \%$.

A conclusão que se pode tirar a partir desse resultado é que, nos países latino-americanos, os agentes econômicos do setor agrícola possuem grandes incentivos a elevar as emissões de poluentes, corroborando com o que foi exposto por Khashman et al. (2016). Tal fato também é visualizado para a economia latino-americana de uma forma geral.

Obteve-se, portanto, uma CKA para cada setor analisado, de maneira que as duas curvas possuem pontos de inflexão diferentes. A partir da análise desses pontos de inflexão é possível obter um indício da dimensão do nível de emissões de $\mathrm{CO}_{2}$ necessárias para que o crescimento econômico atinja a trajetória decrescente na CKA. Nesse caso, obter-se-ia uma medida de custo ambiental do crescimento. Tomando como $P^{*}$ o ponto de inflexão encontrado, a área entre a CKA e o eixo do crescimento no curto prazo pode ser medida como:

$$
e^{*}=\int_{p_{1}}^{P^{*}}(C K A) d\left(\frac{P I B}{P}\right)
$$

onde $p_{1}$ representa o ponto inicial de crescimento da CKA. Já o custo ambiental adicional do crescimento propiciado pela elevação das emissões de $\mathrm{CO}_{2}$ após o ponto de inflexão, pode ser obtido por:

$$
e^{* *}=\int_{p^{*}}^{P^{n}}(C K A) d\left(\frac{P I B}{P}\right)
$$

onde $P^{n}$ representa o ponto final de crescimento obtido na CKA. O custo ambiental diz respeito à área entre a CKA e o eixo das abcissas onde está representado o crescimento, desde o ponto inicial de crescimento obtido até o ponto de inflexão. A área a partir desse ponto até o nível final de crescimento é denominado aqui de custo ambiental adicional. Por ser uma medida de área, esse custo ambiental fornece apenas um indicativo de o quanto se exige em termos de degradação ambiental em decorrência das elevações das emissões de poluentes causadas pelo crescimento econômico. A Tabela 5 demonstra os resultados desse procedimento.

Para as emissões totais, o ponto de inflexão encontrado indica que para que as emissões invertam o comportamento crescente, seria necessário que se 
atingisse um PIB per capita de aproximadamente US\$ 4.243,00. Já em relação à agricultura, esse valor é de US\$266,00. A partir da mensuração do custo ambiental do crescimento para as emissões totais, observa-se que para que uma trajetória decrescente das emissões fosse atingida, seria necessário um montante de 35.854.480 toneladas per capita de $\mathrm{CO}_{2}$, um valor consideravelmente alto. Considerando apenas o setor agrícola, esse valor é de 2.416 .891 toneladas per capita.

Esses valores são ainda maiores para o caso de as economias latino-americanas elevarem seus níveis de crescimento após o ponto de inflexão. Para a economia como um todo, os ganhos de crescimento exigiriam um total de 116.045.060 toneladas per capita de $\mathrm{CO}_{2}$. Já em relação ao setor agrícola, esse valor é de 8.573.236 toneladas per capita de $\mathrm{CO}_{2}$.

O que se pode concluir com o cálculo do custo ambiental obtido por meio da área entre as CKA's e o eixo do crescimento é que esse custo é mais elevado para a economia como um todo, como esperado. O que se observa em ambos os casos é que o custo de elevar o crescimento após o ponto de inflexão é maior do que o custo da elevação do crescimento nos estágios iniciais da CKA. Esse resultado corrobora a ideia de que após um determinado nível de degradação ambiental, os custos ambientais provindos do crescimento econômico se elevam, gerando maiores perdas sociais de bem-estar.

Tabela 6: Estimativas dos custos ambientais do crescimento econômico.

\begin{tabular}{ccc}
\hline & $e^{*}$ & $e^{* *}$ \\
\hline Total & 35.854 .480 & 116.045 .060 \\
Agricultura & 2.416 .891 & 8.573 .236 \\
\hline \multicolumn{3}{c}{ \% da área } \\
\hline Total & 23,60 & 76,40 \\
Agricultura & 21,99 & 78,01 \\
\hline Fonte: Elaborado pelos autores. \\
Nota: Os resultados constam de valores dispostos \\
em toneladas por habitante.
\end{tabular}

Assim, torna-se necessário que os formuladores de políticas públicas voltadas para o contexto ambiental conheçam os impactos ambientais da elevação contínua dos níveis de crescimento e tenham entendimento do ponto ótimo de crescimento de cada setor.

Nesse sentido, as conclusões aqui encontradas indicam a necessidade de implementação de medidas que proporcionem um crescimento econômico com menores impactos ambientais. Reforça-se aqui, portanto, o que foi concluído em estudos como os de Jardón et al. (2017), Martinho (2015) e Almeida et al. (2017).

Assim como foi concluído por Al-Mulali et al. (2015), os resultados encontrados com as CKA's permitem verificar a necessidade de implementação de políticas de incentivo à redução de poluentes como a adequação dos produtores, principalmente do setor agrícola, ao uso de tecnologias com menores impactos ambientais, o que proporcionaria a obtenção de um crescimento econômico com menores impactos ao meio ambiente. 


\section{Considerações finais}

A presente investigação buscou verificar a veracidade da hipótese ambiental de Kuznets para o crescimento econômico total e aplicado ao setor agrícola dos países da América Latina. Para tanto foram utilizados dados dispostos entre 1990 e 2014, os quais foram incorporados em um método capaz de corrigir os problemas provindos da não estacionariedade e da cointegração.

Os resultados encontrados com o método utilizado atestaram a existência de um formato de 'N' para as CKA's relacionadas às emissões totais e às emissões provindas da agricultura. Esses resultados demonstram que, no curto prazo, elevar o crescimento econômico nos dois casos analisados gera aumento dos níveis de degradação ambiental. Com o passar do tempo, essa relação se inverte, de modo que o aumento do crescimento acarretaria em uma redução das emissões de poluentes. Porém, no longo prazo um novo ponto de inflexão é alcançado, de forma que a elevação dos níveis de crescimento econômico proporciona, a partir desse ponto, elevações nas emissões de gases de efeito estufa.

A partir do formato das CKA's, os resultados também permitiram concluir que os agentes econômicos da América Latina estão inseridos em um contexto que os estimulam a elevar as emissões de poluentes em vista do crescimento econômico proporcionado por esse fato, sendo que tal comportamento é mais intenso no setor agrícola.

Por meio do cálculo do custo ambiental das elevações do crescimento, concluiuse que os ganhos de crescimento no longo prazo exigem um maior nível de degradação ambiental se comparado com o curto prazo, seja na agricultura, seja na economia como um todo. Assim, ressalta-se a importância do fato de que os agentes econômicos e os formuladores de políticas ambientais tenham conhecimento dos níveis ótimos de crescimento e emissões de poluentes, para que se possa formular medidas que propiciem um crescimento mais eficiente em termos ambientais.

Por fim, conclui-se que nos países da América Latina existe uma necessidade de implantação de medidas que proporcionem a conciliação do crescimento econômico com a redução da emissão de poluentes, tanto no setor rural quanto na economia como um todo. Constata-se, com isso, a necessidade de utilização de tecnologias e técnicas de trabalho que propiciem uma menor degradação dos recursos naturais.

\section{Referências Bibliográficas}

Al-Mulali, U., Saboori, B. \& Ozturk, I. (2015), 'Investigating the environmental kuznets curve hypothesis in vietnam', Energy Policy 76, 123-131.

Almeida, T. A. N., Cruz, L., Barata, E. \& García-Sánchez, I.-M. (2017), 'Economic growth and environmental impacts: An analysis based on a composite index of environmental damage', Ecological Indicators 76, 119-130.

Apergis, N. \& Ozturk, I. (2015), 'Testing Environmental Kuznets Curve hypothesis in Asian countries', Ecological Indicators 52, 16-22.

Ávila, E. S. \& Diniz, E. M. (2015), 'Evidências sobre Curva Ambiental de Kuznets e convergência das emissões', Estudos Econômicos 45(1), 97-126. 
Ben, M., Ben, S. \& Ozturk, I. (2017), 'Testing environmental Kuznets curve hypothesis: The role of renewable and non-renewable energy consumption and trade in OECD countries', Ecological Indicators 60(2016), 824-831.

Blanco, L., Gonzalez, F. \& Ruiz, I. (2013), 'The impact of FDI on $\mathrm{CO}_{2}$ emissions in Latin America', Oxford Development Studies 41(1), 104-121.

Bueno, R. L. S. (2008), Econometria de séries temporais, São Paulo: Cengage.

CEPAL (2018), 'CEPALSTAT - Bases de Datos y Publicaciones Estadísticas'. URL: http://estadisticas.cepal.org/cepalstat/WEB_CEPALSTAT/estadisti casIndicadores.asp?idiomaē

Choi, I. (2001), 'Unit root tests for panel data', Journal of international money and Finance 20(2), 249-272.

Doan, T. \& Pedroni, P. (2001), 'Purchasing Power Parity Tests In Cointegrated Panels', Review of Economics and Statistics 83, 727-731.

Im, K. S. \& Pesaran, M. H. (2003), 'On the panel unit root tests using nonlinear instrumental variables'.

Jalil, A. \& Mahmud, S. F. (2009), 'Environment Kuznets curve for $\mathrm{CO}_{2}$ emissions: a cointegration analysis for China', Energy policy 37(12), 5167-5172.

Jardón, A., Kuik, O. \& Tol, R. S. (2017), 'Economic growth and carbon dioxide emissions: An analysis of Latin America and the Caribbean', Atmósfera 30(2), 87-100.

Jebli, B. M. \& Youssef, B. S. (2015), 'The environmental Kuznets curve, economic growth, renewable and non-renewable energy, and trade in Tunisia', Renewable and Sustainable Energy Reviews 47, 173-185.

Kang, Y.-Q., Zhao, T. \& Yang, Y.-Y. (2016), 'Environmental Kuznets Curve for $\mathrm{CO}_{2}$ emissions in China: A spatial panel data approach', Ecological indicators 63, 231-239.

Kao, C. \& Chiang, M. (2000), 'On the estimation and inference of a cointegrated regression in panel data', Advances of Econometrics 15, 7-51.

Khashman, A., Khashman, Z. \& Mammadli, S. (2016), 'Arbitration of turkish agricultural policy impact on $\mathrm{CO}_{2}$ emission levels using neural networks', Procedia Computer Science 102, 583-587.

Kuznets, S. (1955), 'Economic growth and income inequality', The American economic review . 45.

Levin, A., Lin, C.-F. \& Chu, C.-S. J. (2002), 'Unit root tests in panel data: asymptotic and finite-sample properties', Journal of econometrics 108(1), 124.

Liu, X., Zhang, S. \& Bae, J. (2017), 'The impact of renewable energy and agriculture on carbon dioxide emissions: investigating the Environmental Kuznets Curve in four selected ASEAN countries', Journal of Cleaner Production 164, 1239-1247. 
Luzzati, T. \& Orsini, M. (2009), 'Investigating the energy-environmental kuznets curve', Energy 34(3), 291-300.

Maddala, G. S. \& Wu, S. (1999), 'A comparative study of unit root tests with panel data and a new simple test', Oxford Bulletin of Economics and statistics 61(S1), 631-652.

Martinho, P. D. (2015), The Agricultural Economics of the 21st Century. New York: Springer.

Nasir, M. \& Rehman, F. U. (2011), 'Environmental Kuznets Curve for carbon emissions in Pakistan: an empirical investigation', Energy Policy 39(3), 18571864.

Page, G., Ridoutt, B. \& Bellotti, B. (2014), 'Location and technology options to reduce environmental impacts from agriculture', Journal of cleaner production 81, 130-136.

Pedroni, P. (1999), 'Critical values for cointegration tests in heterogeneous panels with multiple regressors', Oxford Bulletin of Economics and statistics 61(S1), 653-670.

Pesaran, M. H. (2007), 'A simple panel unit root test in the presence of crosssection dependence', Journal of applied econometrics 22(2), 265-312.

Phillips, P. C. B. \& Moon, H. R. (1999), 'Linear regression limit theory for nonstationary panel data’, Econometrica 67(5), 1057-1111.

Qiao, H., Zheng, F., Jiang, H. \& Dong, K. (2019), 'The greenhouse effect of the agriculture-economic growth-renewable energy nexus: Evidence from G20 countries', Science of The Total Environment 671, 722-731.

Sinha, A. \& Shahbaz, M. (2018), 'Estimation of Environmental Kuznets Curve for $\mathrm{CO}_{2}$ emission: role of renewable energy generation in India', Renewable Energy 119, 703-711.

Tao, S., Zheng, T. \& Lianjun, T. (2008), 'An empirical test of the environmental Kuznets Curve in China: a panel cointegration approach', China Economic Review 19(3), 381-392.

Vlontzos, G., Niavis, S. \& Pardalos, P. (2017), 'Testing for Environmental Kuznets Curve in the EU Agricultural Sector through an Eco-(in) Efficiency Index', Energies 10(12), 1992.

Zafeiriou, E., Sofios, S. \& Partalidou, X. (2017), 'Environmental kuznets curve for eu agriculture: empirical evidence from new entrant eu countries', Environmental Science and Pollution Research 24(18), 15510-15520.

Zakarya, G. Y., Mostefa, B., Abbes, S. M. \& Seghir, G. M. (2015), 'Factors affecting $\mathrm{CO}_{2}$ emissions in the BRICS countries: a panel data analysis', Procedia Economics and Finance 26, 114-125.

Zhang, S., Liu, X. \& Bae, J. (2017), 'Does trade openness affect $\mathrm{CO}_{2}$ emissions: evidence from ten newly industrialized countries?', Environmental Science and Pollution Research 24(21), 17616-17625. 


\section{Apêndice}

Tabela 7: Resultados para o teste de estacionariedade de Levin et al. (2002)

\begin{tabular}{c|c|c|c}
\hline & $\begin{array}{c}\text { Intercepto } \\
\text { individual }\end{array}$ & $\begin{array}{c}\text { Intercepto e } \\
\text { tendência }\end{array}$ & $\begin{array}{c}\text { Sem intercepto e } \\
\text { sem tendência }\end{array}$ \\
\hline \multicolumn{3}{|c}{ Dados gerais } \\
\hline $\ln \left(\frac{E}{P}\right)$ & $5,2518^{*}$ & $-2,2564^{*}$ & 2,5339 \\
$\ln \left(\frac{P I B}{P}\right)$ & $-0,7992$ & $-1,4697$ & 9,9492 \\
$\ln \left(\frac{P P B}{P}\right)^{2}$ & $-0,4305$ & $-1,2849$ & 9,8687 \\
$\ln \left(\frac{P I B}{P}\right)^{3}$ & $-0,0807$ & $-1,0838$ & 9,7902 \\
$\Delta \ln \left(\frac{E}{P}\right)$ & $-11,0303^{*}$ & $-8,5551^{*}$ & $-20,3597^{*}$ \\
$\Delta \ln \left(\frac{P I B}{P}\right)$ & $-12,2116^{*}$ & $-9,8219^{*}$ & $-14,2455^{*}$ \\
$\Delta \ln \left(\frac{P I B}{P}\right)^{2}$ & $-12,2023^{*}$ & $-9,8717^{*}$ & $-14,1731^{*}$ \\
$\Delta \ln \left(\frac{P I B}{P}\right)^{3}$ & $-12,0810^{*}$ & $-9,7126^{*}$ & $-14,1033^{*}$ \\
\hline \multicolumn{4}{|c}{ Dados da agricultura } \\
\hline $\ln \left(\frac{E}{P}\right)$ & $-1,3874$ & 0,9453 & 0,9622 \\
$\ln \left(\frac{P I B}{P}\right)$ & $-1,3244$ & $-1,7639^{*}$ & 3,9527 \\
$\ln \left(\frac{P I B}{P}\right)^{2}$ & $-1,1089$ & $-1,6169$ & 3,8419 \\
$\ln \left(\frac{P I B}{P}\right)^{3}$ & $-0,9062$ & $-1,5107$ & 3,7362 \\
$\Delta \ln \left(\frac{E}{P}\right)$ & $-9,1482^{*}$ & $-5,6423^{*}$ & $-18,9731^{*}$ \\
$\Delta \ln \left(\frac{P I B}{P}\right)$ & $-14,7529^{*}$ & $-12,2966^{*}$ & $-20,5230^{*}$ \\
$\Delta \ln \left(\frac{P I B}{P}\right)^{2}$ & $-14,5820^{*}$ & $-12,3740^{*}$ & $-20,4261^{*}$ \\
$\Delta \ln \left(\frac{P I B}{P}\right)^{3}$ & $-14,5418^{*}$ & $-12,3369^{*}$ & $-20,3364^{*}$ \\
\hline
\end{tabular}

Fonte: Elaborado pelos autores.

Nota: Valores sucedidos do símbolo * denotam a rejeição da hipótese nula de inexistência de estacionariedade a nível de 5\% de significância estatística. 
Tabela 8: Resultados para o teste de estacionariedade $A D F-$ FISHER proposto por Choi (2001)

\begin{tabular}{c|c|c|c}
\hline & $\begin{array}{c}\text { Intercepto } \\
\text { individual }\end{array}$ & $\begin{array}{c}\text { Intercepto e } \\
\text { tendência }\end{array}$ & $\begin{array}{c}\text { Sem intercepto e } \\
\text { sem tendência }\end{array}$ \\
\hline \multicolumn{3}{c}{ Dados gerais } \\
\hline $\ln \left(\frac{E}{P}\right)$ & $88,4188^{*}$ & 64,7248 & 67,8541 \\
$\ln \left(\frac{P I B}{P}\right)$ & 34,9700 & 77,2857 & 4,7380 \\
$\ln \left(\frac{P I B}{P}\right)^{2}$ & 33,2537 & 75,3286 & 4,8524 \\
$\ln \left(\frac{P I B}{P}\right)^{3}$ & 31,6652 & 73,1579 & 4,9731 \\
$\Delta \ln \left(\frac{E}{P}\right)$ & $372,1180^{*}$ & $330,6710^{*}$ & $486,2720^{*}$ \\
$\Delta \ln \left(\frac{P I B}{P}\right)$ & $303,2530^{*}$ & $230,0260^{*}$ & $343,4420^{*}$ \\
$\Delta \ln \left(\frac{P I B}{P}\right)^{2}$ & $300,9690^{*}$ & $228,3320^{*}$ & $340,7410^{*}$ \\
$\Delta \ln \left(\frac{P I B}{P}\right)^{3}$ & $298,6350^{*}$ & $226,7500^{*}$ & $338,1490^{*}$ \\
\hline & \multicolumn{3}{c}{ Dados da agricultura } \\
\hline $\ln \left(\frac{E}{P}\right)$ & 58,9884 & 55,8473 & 41,1289 \\
$\ln \left(\frac{P I B}{P}\right)$ & 49,1672 & 66,9049 & 30,7995 \\
$\ln \left(\frac{P I B}{P}\right)^{2}$ & 48,2833 & 65,8993 & 31,7636 \\
$\ln \left(\frac{P I B}{P}\right)^{3}$ & 47,5437 & 65,1748 & 32,6934 \\
$\Delta \ln \left(\frac{E}{P}\right)$ & $313,1870^{*}$ & $233,1500^{*}$ & $431,4700^{*}$ \\
$\Delta \ln \left(\frac{P I B}{P}\right)$ & $362,5120^{*}$ & $296,5650^{*}$ & $463,5490^{*}$ \\
$\Delta \ln \left(\frac{P I B}{P}\right)^{2}$ & $360,0470^{*}$ & $293,8920^{*}$ & $459,6770^{*}$ \\
$\Delta \ln \left(\frac{P I B}{P}\right)^{3}$ & $356,2770^{*}$ & $291,5870^{*}$ & $456,1280^{*}$ \\
\hline
\end{tabular}

Fonte: Elaborado pelos autores.

Nota: Valores sucedidos do símbolo * denotam a rejeição da hipótese nula de inexistência de estacionariedade a nível de 5\% de significância estatística. 